\title{
ABDOMINAL AORTIC PSEUDOANEURYSM ASSOCIATED WITH A METASTATIC GERM CELL TUMOR: A RARE COMPLICATION
}

\author{
Antonio Eduardo Zerati ${ }^{1}$, Kenji Nishinari ${ }^{1}$, Guilherme Yazbek $^{1}$, Nelson Wolosker ${ }^{2}$, Francisco Paulo da Fonseca ${ }^{3}$
}

\section{INTRODUCTION}

The first description of en bloc resection of malignant neoplasia involving the abdominal aorta along with its reconstruction was published by Crawford and DeBakey ${ }^{1}$ in 1956. Since then, few studies have been published, and most of the aortic reconstructions have been associated with retroperitoneal tumors originating from germinative cells., ${ }^{2,3}$ On rare occasions, these tumors cause erosion of the aorta due to tumor necrosis that follows chemotherapy, with the formation of a pseudoaneurysm, and only 2 cases have been described. ${ }^{4,5}$

We describe the case of a patient with primary testicular cancer who presented retroperitoneal recurrence with involvement of the aorta and formation of a pseudoaneurysm during the follow-up, and underwent surgical treatment.

\section{CASE DESCRIPTION}

The patient was a 35-year-old white male who had undergone radical orchiectomy 8 years earlier due to embryonic carcinoma. One year before coming to us, he started to experience pain in his flank and left lumbar region.

Magnetic resonance imaging (MRI) of the abdomen was performed, and this detected an extensive retroperitoneal mass that involved the abdominal aorta, intercavoaortic space, and left kidney. The patient initially received chemotherapy consisting of 4 cycles of cisplatin, etoposide, and bleomycin and 4 cycles of paclitaxel, ifosfamide, and cisplatin. The subsequent control MRI showed that there had been a $50 \%$ reduction in the mass and a focal dilatation of the aorta in the segment surrounded by the tumor. Magnetic resonance angiography showed a pseudo-

1. Department of Vascular Surgery and Endovascular, Hospital do Câncer A.C. Camargo, São Paulo, Brazil.

2. Department of Vascular and Endovascular Surgery, Hospital das Clínicas School of Medicine, University of São Paulo, São Paulo, Brazil.

3.Hospital Einstein, São Paulo, Brazil

Email: aezerati@uol.com.br aneurysm of the infrarenal aorta that was $3 \mathrm{~cm}$ in diameter (Figure 1). The tumor marker findings were $\alpha$ fetoprotein $=6.1 \mathrm{ng} / \mathrm{mL}$ and $\beta$-human chorionic gonadotrophin $<2 \mathrm{mIU} / \mathrm{L}$.

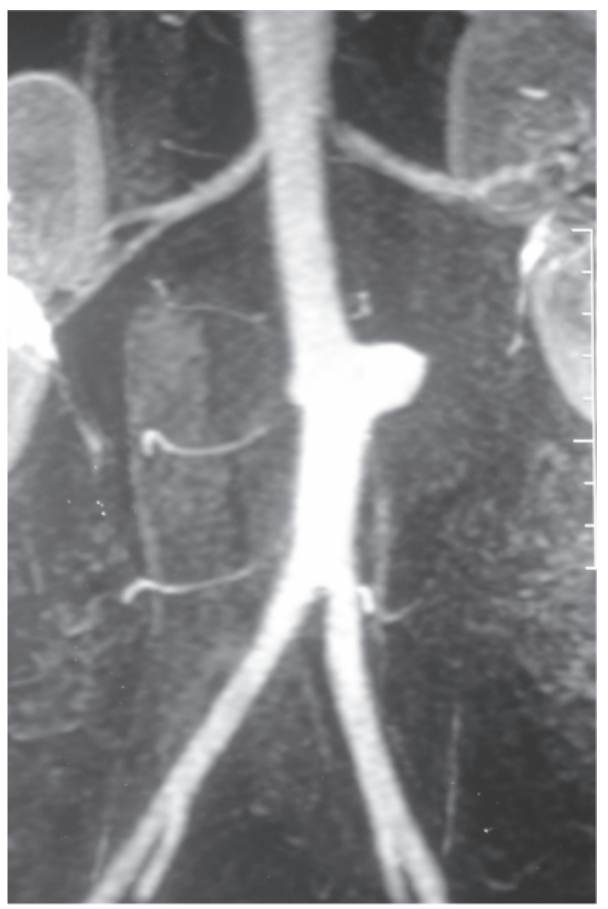

Figure 1 - Preoperative magnetic resonance angiography showing the aortic pseudoaneurysm.

Surgical treatment was proposed because of the presence of the pseudoaneurysm and the residual mass. The tumor was identified by means of median laparotomy, and control of the vascular structures was done (juxtarenal aorta, renal, and iliac arteries). Dissection of the vena cava was not necessary, because there was no invasion of this vessel, which was only pushed by the tumor. This was followed by dissection around the tumoral mass. After endovenous administration of heparin, clamping and sectioning of the vessels was performed as the last stage of the en bloc tumoral resection, which involved the aorta with the 
pseudoaneurysm (Figure 2), retroperitoneal lymph nodes, and the left kidney and adrenal.

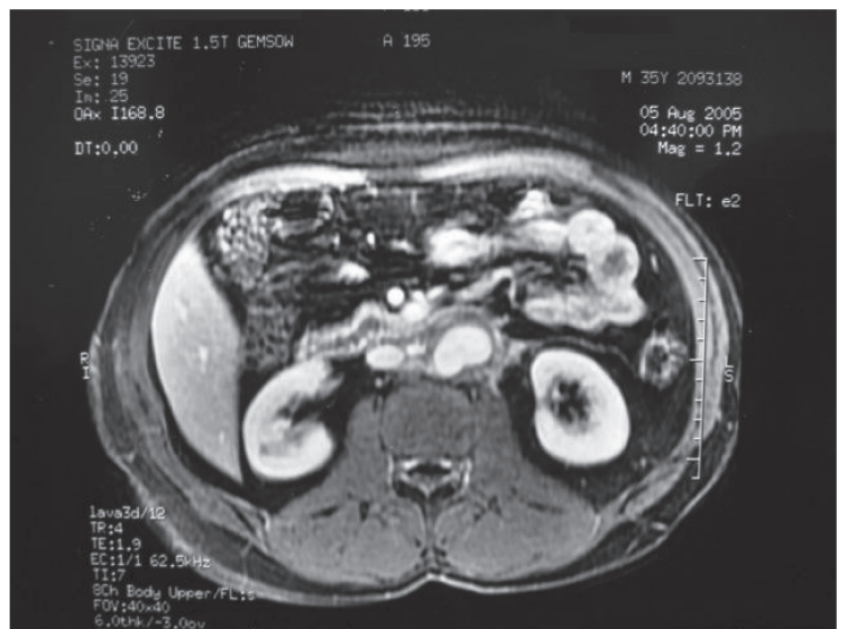

Figure 2 - Preoperative magnetic resonance angiography showing the aortic pseudoaneurysm-transversal section.

Vascular reconstruction was performed by means of aortobiiliac grafting with end-to-end anastomoses, using a Dacron 16 × $8 \mathrm{~mm}$ prosthesis. The duration of vascular clamping was 50 minutes, and the operation took 5 hours in total. Anatomopathological examination showed the presence of fibrotic tissue with areas of necrosis and calcification, without evidence of residual neoplasia.

The postoperative period was uneventful, and the patient was discharged from the hospital after 8 days. After 1 year of follow-up, the patient was found to be asymptomatic from the oncological and vascular points of view. Complementary tests showed that the retroperitoneal mass was absent, and the tumor markers were normal. Magnetic resonance angiography showed that the vascular graft was patent and free from complications (Figure 3).

\section{DISCUSSION}

Patients with retroperitoneal tumors originating in nonseminomatous germinative cells are initially treated by means of chemotherapy. ${ }^{2,3}$ However, subsequent surgical treatment may be necessary for resecting the residual mass, ${ }^{2-4}$ since there are no preoperative tests that would indicate with certainty whether these lesions are composed of fibrotic tissue alone, or whether they also contain malignant cells.

Suspected aortic involvement is usually detected preoperatively. In such cases, imaging tests show that there is no well-defined cleavage plane between the tumoral mass and the aortic wall. Nevertheless, real verification of vascular invasion is only done intraoperatively. At this time,

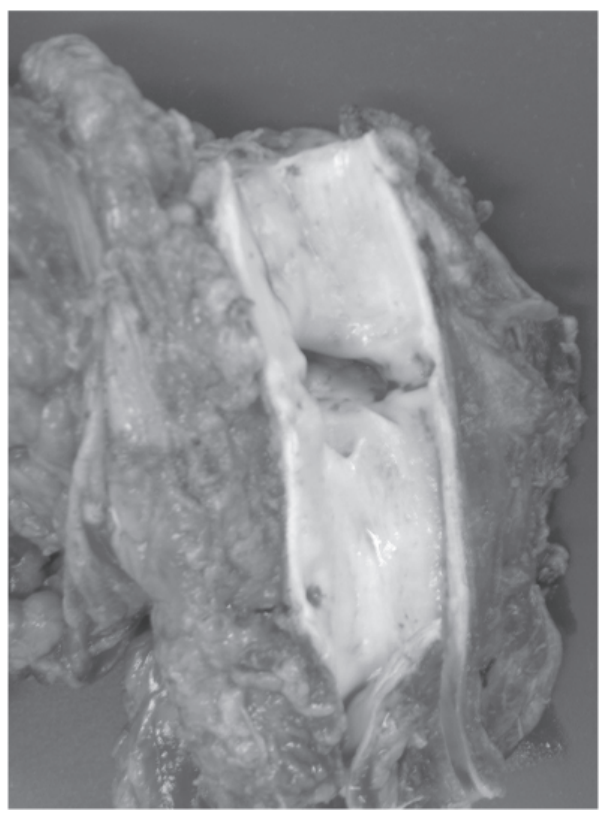

Figure 3 - Fragment from the tumoral bloc showing a segment of the aorta with wall erosion.

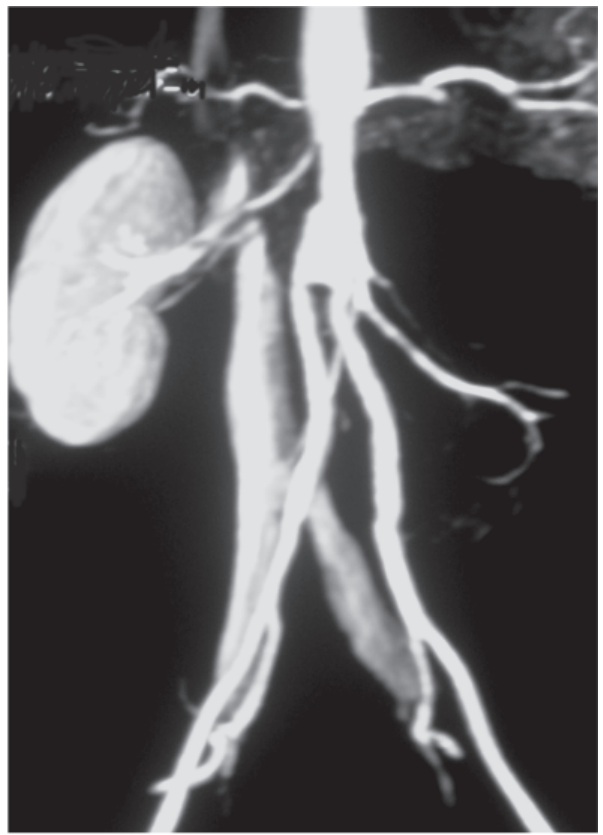

Figure 4 - Postoperative magnetic resonance angiography showing the aortobiiliac graft, without abnormalities.

tumor resection can be performed by means of 2 techniques: in association with subadventitial dissection of the aorta or in association with its resection and reconstruction.

If subadventitial dissection of the aorta is performed, there is no risk of tissue ischemia, although some serious complications may be related to this technique. During the 
operation, because of the absence of a well-defined cleavage plane between the aorta and the mass, there may be parietal lacerations ${ }^{6}$ that require immediate correction. Following the operation, because of the parietal thinning that is inherent to the technique, the aorta may rupture ${ }^{7,8}$ or an aortoenteric fistula may develop, ${ }^{11}$ which may lead to death or the need for a vascular grafting as a matter of urgency.

In some cases to avoid tumor violation, en bloc resection with the aorta is necessary, followed by vascular reconstruction by means of anatomical grafting using a prosthesis. ${ }^{2,3}$ Utilization of this technique makes it possible to obtain adequate oncological safety margins and involves a small risk of vascular complications, such as compartment syndrome in the lower limbs. ${ }^{4}$

Abdominal aortic pseudoaneurysm secondary to invasion by malignant tumors is very rare, and only 2 cases other have been described. ${ }^{7,8}$ Terry et $\mathrm{al}^{7}$ described a 28 year-old patient who had a testicular tumor and retroperitoneal mass with involvement of the aorta. The patient underwent orchiectomy followed by chemotherapy. A large aortic pseudoaneurysm formed, which progressed to rupture and was treated by means of an aortic endoprosthesis as a matter of urgency. Subsequently, the patient underwent a further 2 cycles of chemotherapy and then resection of the residual mass. After 1 year of follow-up, the patient was found to be asymptomatic, and aortography showed that the endoprosthesis did not present any complications. Jerius et $\mathrm{al}^{8}$ reported the case of a 20 -year-old patient with a testicular tumor and retroperitoneal mass with involvement of the aorta. The patient underwent orchiectomy followed by chemotherapy, and also progressed with the formation of a large aortic pseudoaneurysm. Elective en bloc resection was performed, followed by aortic reconstruction using a Dacron prosthesis, using a temporary bypass ${ }^{99}$ to decrease the duration of ischemia in the lower limbs. After 7 months of follow-up, the patient was found to be asymptomatic.

In the present case, the patient presented neoplasia with an aortic complication that was similar to what had previously been described. The pseudoaneurysm is attributed to tumor necrosis, including the cells within the aortic wall, secondary to chemotherapy. Surgical treatment in the present case was indicated for 2 reasons: first, because of the presence of the pseudoaneurysm, with its risks of serious complications such as enlargement, rupture, acute thrombosis, and distal embolization, and second, because of the presence of a large residual mass. During the operation, it was possible to control the vascular structures proximally and distally to the tumoral bloc, and classical anatomical reconstruction was performed using a Dacron prosthesis, which progressed without recurrence. We did not use the temporary bypass because the tumor was not violated, reducing the risk of rupture of the pseudoaneurysm; in addition, the clamping of arteries occurred only after the tumor was ready to be resected, avoiding a long period of ischemia. We use the temporary bypass only when arterial clamping is necessary before the complete dissection of the mass, because in this case, the time of clamping is unknown. The time of cross-clamping in our case (50 minutes) is unlikely to cause compartment syndrome.

We have described a rare case of a patient with a retroperitoneal tumor that was associated with an aortic pseudoaneurysm, and who underwent surgical treatment with good results from the initial follow-up.

\section{REFERENCES}

1. Crawford ES, DeBakey ME. Wide excision including involved aorta and vena cava and replacement with aortic homograft for retroperitoneal malignant tumors: report of two cases. Cancer. 1956;9:1085-91.

2. Kelly R, Skinner D, Yellin AE, Weaver FA. En bloc aortic resection for bulky metastatic germ cell tumors. J Urol. 1995;153:1849-51.
3. DiPerna CA, Bowdish ME, Weaver FA, Bremner RM, Jabbour N, Skinner D, et al. Concomitant vascular procedures for malignancies with vascular invasion. Arch Surg. 2002;137:901-7.

4. Terry PJ, Houser EE, Rivera FJ, Palmaz JC, Sarosdy MF. Percutaneous aortic stent placement for life threatening aortic rupture due to metastatic germ cell tumor. J Urol. 1995;153:1631-4. 
5. Jerius JT, Elmajian DA, Rimmer DM, Spires KS. Floppy aortic graft reconstruction for germ cell tumor invasion of the infrarenal aorta. $\mathrm{J}$ Vasc Surg. 2003;37:889-91.

6. Morin JF, Prova MB, Jewett MAS, Ameli FM. Vascular injury and repair associated with retroperitoneal lymphadenectomy for nonseminomatous germinal cell tumours of the testis. Can J Surg. 1992;35:253-6.

7. Jaeger N, Weissbach L, Hartlapp JH, Vahlensieck W. Risk/benefit of treating retroperitoneal teratoid bulky tumors. Urology. 1989;34:14-7.
8. Donohue JP, Thornhill JA, Foster RS, Bihrle R. Vascular considerations in postchemotherapy. Retroperitoneal lymph-node dissection: Part II. World J Urol. 1994;12:187-9.

9. Nishinari K, Wolosker N. Regarding "Floppy aortic graft reconstruction for germ cell tumor invasion of the infrarenal aorta". J Vasc Surg. 2004;39:922. 